\title{
Ausfallbürge der Mehrheitsgesellschaft
}

\author{
Stand und Entwicklungsperspektiven von \\ sozialen Diensten durch muslimische Organisationen
}

Yunus Ulusoy

Yunus Ulusoy ist Leiter der

Abteilung »Ethnische Ökonomie, Modellprojekte« bei der Stiftung Zentrum für Türkeistudien in Essen. Internet http://www.zft-online.de
Die muslimischen Organisationen können ibre Existenz und ihre Geltungsansprüche nicht theologisch rechtfertigen, sondern durch die praktische Akzeptanz und Frequentierung ibrer Einrichtungen durch die gläubigen Muslime. Insofern besitzen die sozialen und religiösen Dienste eine zentrale Bedeutung für die Akzeptanz der Moscheevereine durch die gläubigen Muslime. In diesem Kontext steigen die Erwartungen der Mehrheitsgesellschaft und der Muslime an die muslimischen Organisationen.

Die sozialpolitischen Prinzipien des Islam lassen sich in drei Kategorien zusammenfassen: Gleichheit, Ausgleich und Gemeinschaft. Das Gleichheitsprinzip besagt, dass sich der Stellenwert des Gläubigen nicht nach Rasse, Geschlecht, Vermögen oder sozialer Schichtzugehörigkeit, sondern nach der Einhaltung der religiösen Gebote- und Verbote definiert. Vor Gott sind somit alle Gläubigen gleich. Das Gemeinschaftsprinzip bringt die Idee der Einheit und Einigkeit der Muslime zum Ausdruck. Das Ausgleichsprinzip will die individuellen Bedürfnisse mit den Interessen des Gemeinwohls in Einklang bringen. Die sozialpolitischen Prinzipien finden ihre Begründung u. a. im Glaubensbekenntnis, im rituellen Gebet, im Fasten, in der Armensteuer und der Pilgerfahrt als Grundsäulen des Islam.

Die sozialpolitischen Ziele im Islam werden durch eine Reihe von sozialpolitischen Instrumenten umgesetzt. $\mathrm{Zu}$ den muslimischen Ethik- und Moralvorstellungen gehört, dass alle für ihr unmittelbares Umfeld Verantwortung tragen: Eltern für ihre Kinder und Kinder für ihre Eltern, Verwandte und Nachbarn füreinander, der Einzelne für die Gesellschaft und umgekehrt. In diesem Kontext kommt der privaten Verantwortung die entscheidende Rolle zu. Der Staat kann flankierend die Rahmenbedingungen setzen und für diejenigen sorgen, die aus der sozialen Solidarität herausfallen.

Die Pflichten betreffen in erster Linie den Gläubigen, nicht das System. Es gibt somit keine unmittelbare Vorgabe für ein sozialpolitisches System. Die Bandbreite kann eine starke private und soziale Verantwortung des Einzelnen oder der Gemeinschaft ohne starke staatliche Intervention bis hin zu einer umfassenden staatlichen Intervention zur Regulierung der sozialen Absicherung seiner Bürger umfassen. In der Praxis der muslimischen Länder, insbesondere der Herkunftsländer der muslimischen Migranten in Deutschland, gibt es keine umfassende staatliche Absicherung.

\section{Eine Bestandsaufnahme der sozialen Dienste für Muslime in Deutschland}

Die soziale Versorgung der rund 3,4 Millionen Muslime in Deutschland ist bislang unzureichend, da die kirchlichen, kommunalen und freien Träger von sozialen Diensten oft nicht über die nötige kulturspezifische und personelle Kompetenz verfügen, um die Bedürfnisse der Muslime zu kennen und durch spezifische Angebote hierauf reagieren zu können. Vom Prinzip her müssten alle sozialen Dienste, die durch staatliche Zuschüsse finanziert werden, für alle Gruppen offen stehen. Das ist im Bereich der kirchlichen sozialen Aktivitäten nicht überall gegeben. Die Muslime können das katholische oder evangelische Krankenhaus in Anspruch nehmen, aber nicht immer den kirchlichen Kindergarten.

In den ersten Jahren der Migrationsgeschichte konzentrierte sich der soziale Dienst um die Lösung von Alltagsproblemen, die in erster Linie aufgrund der fehlenden Sprachkenntnisse entstanden. Heute benötigt man soziale Dienste für alle Altersgruppen und alle Lebensbereiche. Insofern gibt es eine sozialpolitische Schieflage in Deutschland. Eine Bevölkerungsgruppe, die aufgrund der migrationsspezifischen Lebensumstände stärker eine soziale Betreuung benötigt als im Herkunftsland, kann bestimmte Angebote des Wohlfahrtsstaates nicht in Anspruch nehmen, weil sie 
eine einseitige religiöse Orientierung aufweisen oder weil die Angebote, ihre Bedürfnisse nicht ausreichend abdecken.

Zwischen den muslimischen Selbsthilfeangeboten und den Angeboten der deutschen Wohlfahrtspflege liegt der entscheidende Unterschied darin, dass die deutschen Angebote nicht als »eigene soziale Dienste « angesehen werden, sondern als fremde Angebote, auf die man im Notfall zurückgreift. Die Angebote der Moscheevereine (vgl. Kasten »Was Moscheevereine heute leisten «) werden ausschließlich oder ergänzend zu denen der Mehrheitsgesellschaft organisiert und durchgeführt. Häufig bekommt die Mehrheitsgesellschaft nicht mit, welche sozialen Dienste bereits als Selbsthilfe angeboten werden. Dennoch können auch muslimische Organisationen die Muslime nicht optimal versorgen, da sie häufig mit strukturellen, personellen und finanziellen Engpässen zu kämpfen haben. Die sozialen Dienste können somit nicht immer vereinsintern etabliert und kontinuierlich angeboten werden. Die Mitgliedsbeiträge und Spenden werden derzeit für den Bau und Unterhalt der Moscheen verwendet. Da die Gemeinden erst in den letzten beiden Jahrzehnten entstanden sind, orientiert sich noch der Schwerpunkt der Arbeit auf den Aufbau der Infrastruktur für die religiösen Dienste. Selbst diese religiöse Infrastrukturarbeit kann von vielen Gemeinden nicht mit eigenen Mitteln kurzfristig finanziert werden, so dass sie Bankkredite aufnehmen müssen. Viele örtliche Gemeinden sind derzeit damit beschäftigt, die Finanzierung dieser religiösen Infrastruktur sicher zu stellen. Hinzu kommt, dass ein beträchtlicher Teil der Gemeindeangehörigen heute als Arbeitslose und Rentner mit begrenztem Einkommen keine Möglichkeit hat, die Arbeit der eigenen Moscheegemeinde finanziell zu unterstützen.

Die zunehmende Armut unter den Gemeindeangehörigen erschwert daher die Möglichkeiten der Moschee, soziale Dienste anzubieten und sie zu finanzieren. Gleichzeitig steigt aufgrund des Anteils von Gemeindeangehörigen, die im Arbeitsmarkt nicht integriert sind, die Notwendigkeit für diese Menschen soziale Dienste anzubieten. Erschwerend kommt hinzu, dass in den Familien die sozialenund generationsbedingten Konflikte zwischen Eltern und Kindern migrationsbedingt verstärkt werden. Eltern orientieren sich zum Teil an Werte und Moralvorstel- lungen, die aus dem Herkunftsland stammen und hier konserviert an die Kinder weitervermittelt werden. Die Kinder hingegen erfahren eine »deutsche « Sozialisation im Schulsystem, ohne dass sie die entsprechende familiäre Unterstützung einer deutschen Familie im Hintergrund haben. Aufgrund dieser Konfliktlagen müssten die Moscheegemeinden, Sozialarbeiter beschäftigen und Familien-, Erziehungs- und Konfliktberatungszentren aufbauen. Ohne eine staatliche Unterstützung wäre eine solche Infrastruktur von den Moscheen nicht finanzierbar, unabhängig davon, ob die Mehrheitsgesellschaft solche sozialen Dienste der Mocheegemeinden integrationspolitisch gut heißt oder nicht.

\section{Bewertung der sozialen Dienste von muslimischen Selbstorganisationen}

Soziale und karitative Dienste runden heute das religiöse Angebot der Moscheevereine $\mathrm{ab}$, wenn hierfür zusätzliche organisatorische, finanzielle und ehrenamtliche Kapazitäten zur Verfügung stehen. Dieser positive externe Effekt der muslimischen Organisationen wird von der Mehrheitsgesellschaft entweder nicht erkannt, oder durch die Vorstellung überlagert, in den Moscheen werde eine integrationshemmende Indoktrination der Muslime betrieben. Die muslimischen Selbstorganisationen haben ein Eigeninteresse, ihre sozialen Dienste weiter zu entfalten. Denn ihre Akzeptanz bei den Muslimen, und damit auch ihre Rechtfertigung in der Öffentlich- keit, können sie nur dadurch erlangen, dass sie der migrationsspezifischen Mangelsituation zur Befriedigung von sozialen, gesellschaftlichen und insbesondere religiösen Bedürfnissen der Muslime spezifische Angebote entgegensetzen.

Ein Teil der politischen und gesellschaftlichen Akteure der Mehrheitsgesellschaft aber auch der eigenen ethnischen Gruppe betrachten diese zunehmende Bedeutung der Moscheevereine mit Misstrauen bis hin zur offenen Ablehnung.

Es ist festzustellen, dass es in der Praxis nicht die sozialpolitischen Entwicklungen und soziale Dienste des Islam in Deutschland gibt, sondern sozialpolitische Ziele und Angebote von muslimischen Selbstorganisationen als Grundlagen ihrer Daseinsberechtigung. Somit werden sich in Deutschland keine Wohlfahrtsorganisationen mit islamimmanenter Legitimation parallel zu den kirchlichen Strukturen entwickeln können, sondern sich ergänzende und gegebenenfalls miteinander konkurrierende soziale Dienste von muslimischen Selbstorganisationen.

Es ist nicht zu erwarten, dass die verschiedenen muslimischen Verbände sich im Bereich der sozialen Dienste einigen und eine gemeinsame muslimische Wohlfahrtsorganisation in absehbarer Zeit aufbauen. Die sozialen Dienste von gesellschaftlichen Gruppen wie Kirchen und Vereinen entlasten den Staat, selbst wenn er diese mitunterstützt. Deshalb sollten anhand klarer und transparenter Kriterien integrationsfördernde Angebote von muslimischen Selbstorganisationen von staatlichen Stellen mitunterstützt werden.

\section{Was Moscheevereine heute leisten}

Die Moscheevereine in Deutschland bieten heute neben den originären religiösen Angeboten eine breite Palette von Diensten an:

- Seelsorgerische Tätigkeiten, beispielsweise Gefängnisseelsorge, Krankenseelsorge, religiöse Trauungen und religiöse Hochzeitszeremonien

- Sozialarbeit, beispielsweise Kinderbetreuung, familiäre Konfliktberatung, Frauenund Jugendarbeit, Seniorenarbeit, Behördengänge, Übersetzungshilfen und Informationsveranstaltungen mit deutschen Netzwerkpartnern

- Bildungs- und Weiterbildungsangebote, beispielsweise Alphabetisierungskurse, Deutschkurse, Qualifizierungsangebote, Nachhilfeunterricht, Informationsveranstaltungen über das Schul- und Ausbildungssystem, Muttersprachkurse, Bildungsreisen und Computerkurse

- Freizeit- und Sportangebote, beispielsweise Teestuben als soziale Begegnungsstätten, Jugendzentren, mystische Musik, Folkloregruppen und Fußball- und andere Sportarten als Vereins- und Freizeitsport

- Integrationsdienste, beispielsweise Tage der offenen Moschee, interreligiöse Begegnungen und Nachbarschaftsfeste

- Wohlfahrtsarbeit, beispielsweise Stipendien, Studienbeihilfen, Hilfskampagnen, Moscheebauten und Bestattungs- und Heimatüberführungsfonds Yunus Ulusoy 